\title{
A survey of management of congenital nasolacrimal duct obstruction by pediatric primary health care providers in Spain
}

\author{
Alicia Galindo-Ferreiro ${ }^{1,2}$, Teresa Palencia-Ercilla ${ }^{3}$, Livia Mendoza Ferreira ${ }^{4}$, Alberto Galvez-Ruiz ${ }^{1}$, Denise C.M. Zornoff ${ }^{5}$, \\ Rajiv Khandekar6, Silvana A. Schellini ${ }^{4}$ \\ ${ }^{1}$ King Khaled Eye Specialist Hospital, Riyadh - Saudi Arabia \\ ${ }^{2}$ Department of Ophthalmology, Complejo Asistencial de Palencia, Palencia - Spain \\ ${ }^{3}$ Primary Health Care Pediatric Center Cuellar, Segovia - Spain \\ ${ }^{4}$ Department of Ophthalmology, Faculdade de Medicina de Botucatu-UNESP, São Paulo - Brazil \\ ${ }^{5}$ Distance Education and Health Information Technology Center, Faculdade de Medicina de Botucatu-UNESP, Sao Paulo - Brazil \\ ${ }^{6}$ Diagnostic Research, King Khaled Eye Specialist Hospital, Riyadh - Saudi Arabia
}

\begin{abstract}
Purpose: To survey the management of congenital nasolacrimal duct obstruction (CNLDO) by pediatric primary health care providers in Spain.

Methods: This was a descriptive study using a web-based questionnaire to evaluate the perceptions of the members of the Pediatric Primary Care Society in Castilla-León, Spain (APAPCYL), regarding management of CNLDO. The questionnaire contained 14 direct questions and was sent by e-mail to all the pediatricians. All the responses were analyzed by the frequency of occurrence and percentages.

Results: Ninety physicians responded to the questionnaire. Massage 2 or 3 times a day was the initial treatment advised by $60.47 \%$ of pediatricians. Nearly half of the pediatricians recommended continuing massage until symptoms resolved. Fewer than $50 \%$ of children required referral to an ophthalmologist. Reasons for an ophthalmic consult included persistence of symptoms among $87.21 \%$ of pediatricians and parental/guardian request among $10.5 \%$ of pediatricians. According to $45.6 \%$ of pediatricians, their knowledge about CNLDO is limited, and $92.2 \%$ would like to receive further training on CNLDO.

Conclusions: Massage was the main initial treatment for managing CNLDO among pediatricians. The outcomes of this survey indicated that massage fails in fewer than $50 \%$ of patients and an ophthalmic referral is required for these cases.
\end{abstract}

Keywords: Congenital, Lacrimal disease, Massage, Nasolacrimal duct obstruction, Pediatrician

\section{Introduction}

Congenital nasolacrimal duct obstruction (CNLDO) due to an imperforate membrane at Hasner valve is the most common abnormality of the lacrimal system in childhood $(1,2)$. The incidence of CNLDO varies from $1.75 \%$ (1) to $6 \%-12 \%(3)$ to $20 \%-30 \%(4,5)$ of normal newborns $(6)$. Only

\section{Accepted: November 13, 2016}

Published online: December 20, 2016

Corresponding author:

Alicia Galindo-Ferreiro

Oculoplasty Department

King Khaled Eye Specialist Hospital

Aruba Road

POB 7191

11462, Riyadh

Saudi Arabia

ali_galindo@yahoo.es
$1 \%-6 \%$ of these children become symptomatic, presenting with epiphora (4).

Primary care pediatricians are key to the overall health of newborn patients and children (7-9). In cases with ocular manifestations, pediatrician often adopt an interdisciplinary approach, working closely with ophthalmologists.

Although most cases of CNLDO resolve spontaneously within the first year of life, some may persist and cause significant distress to the child, parents, and pediatrician (2, $10,11)$. Thus, a proper therapeutic strategy for CNLDO is essential for primary care providers.

The medical management of CNLDO remains controversial, especially the type of massage, time to stop massage, and the best moment to refer the patient to an ophthalmologist for probing or further treatment $(1,10)$.

Ophthalmologists tend to receive complex CNLDO cases and likely do not have data on the number of cases that resolved spontaneously.

This study evaluated the treatments for CNLDO recommended by the members of the Pediatric Primary Care 
Society in Castilla-León, Spain (APAPCYL). To our knowledge, this is the first study evaluating the perceptions of CNLDO among primary care pediatricians.

\section{Methods}

A qualitative web-based survey was conducted targeting 212 members of APAPCYL from May 2016 to June 2016. Institutional review board approval was deemed unnecessary. The target population was general pediatricians working in urban and rural areas of Castilla-Leon, Spain. This study adhered to the tenets of the Declaration of Helsinki. The diagnosis of CNLDO at the primary health care level is based on clinical signs, characterized by the presence of epiphora with a high tear meniscus, mucopurulent discharge, and crusting of the eyelashes (12).

Pediatricians received an invitation to participate in the research by e-mail. To improve the participation rate, a reminder request was sent after 2 weeks. The online survey contained 14 multiple-choice questions and was created at Lyme Survey open source software, configured in an adaptive way, progressively using follow-up questions or spaces for comments, based on the respondent's answers.

The survey was accessible at www3.fmb.unesp.br/ questionarios/index.php/852412/lang-es and evaluated pediatrician attitudes of the treatments for CNLDO (Appen$\operatorname{dix} A$, available online), including demographic information, length of practice, experience with treatment of CNLDO, and the number of children treated for CNLDO. Questions on CNLDO management focused on medical treatment during the first year of life, ophthalmologist referral, and willingness of pediatricians to be trained on lacrimal drainage.

All primary questions were mandatory, hence answers were required to continue with the survey.

The survey was anonymous and, to ensure that responses could not be linked to the respondents, the Internet protocol address (an automatic numerical value allocated to every computer connected to the web) was removed from individual responses at the time of analysis.

\section{Statistical analysis}

The responses were entered in an Excel spreadsheet (Microsoft, Redmond, WA, USA) and analyzed according to frequency.

\section{Results}

Ninety questionnaires were returned, which accounts for $42.45 \%$ of all of general pediatricians in the region. Fifty-three (59\%) pediatricians had practiced for more than 15 years and 71 (78.9\%) pediatricians had seen more than 10 cases of CNLDO over their clinical career.

Eighty-six (95.55\%) of the pediatricians diagnosed CNLDO clinically, and a confirmatory diagnostic test was not requested by 89 (99\%) of the pediatricians. Forty-four (49\%) pediatricians diagnosed the condition within the $1^{\text {st }}$ week to the $1^{\text {st }}$ month of the child's life and 39 (43.3\%) diagnosed CNLDO in children 2 months to 6 months of age.
Eighty-six (95.5\%) pediatricians recommended conservative massage as the initial treatment. The frequency of massage was 2 to 3 times a day among 54 (60.4\%) pediatricians.

Thirty-nine $(47.6 \%)$ pediatricians recommended continuing massage until the symptoms disappeared, 12 (14.6\%) pediatricians advised massage until 12 months of age, whereas 30 (36.6\%) recommended massage until 6 months of age. Fifty-eight $(67.4 \%)$ pediatricians thought that a child should respond positively to massage therapy within 6 months, and 28 (32.6\%) pediatricians responded that CNLDO is cured by massage for 6 to 12 months.

Fewer than $50 \%$ of CNLDO cases warranted ophthalmologist referral because the condition resolved with massage. Reasons for ophthalmic referral included the following: 71 (87.2\%) pediatricians thought the child would be better treated by an eye specialist and 9 (10.5\%) pediatricians referred based on parent/guardian request.

Forty-one $(45.6 \%)$ pediatricians considered their knowledge of CNLDO limited, and 40 (45.6\%) considered it fair. Thirty-two (35\%) pediatricians received training on CNLDO during their residency program.

Sixty $(66.7 \%)$ pediatricians graded their diagnosis of CNLDO as good and 51 (56.7\%) pediatricians graded their treatment of CNLDO as good.

Eighty-two (92.2\%) pediatricians expressed an interest in further training for CNLDO.

\section{Discussion}

Pediatricians are often the first medical practitioners dealing with CNLDO. Hence, the aim of this survey was to evaluate their opinions regarding their knowledge of diagnosis and management of CNLDO.

We found 3 surveys on CNLDO in the literature: 1 surveyed general ophthalmologists in the United Kingdom (3), another surveyed Latin American oculoplastic surgeons (10), and 1 reported on pediatric ophthalmologists in the United States (13). To our knowledge, this is the first survey of CNLDO enrolling primary care pediatricians.

The outcomes of the current survey indicate that pediatricians manage CNLDO appropriately and refer to an ophthalmologist if the patient requires further assessment and management.

Including stillborn births, $54.3 \%$ (6) to $70 \%$ (14) of neonates present with CNLDO at delivery. However, only $1 \%-6 \%$ of these children become symptomatic (4). These symptomatic cases are identified and managed by primary care pediatricians.

Almost all pediatricians who participated in the current survey regularly treat children with CNLDO and had experience in dealing with this condition. However, only $35 \%$ received training on CNLDO during their residency.

Approximately half of the responders (50.57\%) diagnosed CNLDO within the 1st week to 1st month of the child's life, which coincides with the onset of tear production (1 to 2 weeks after birth) $(2,4,11,15,16)$.

Evaluation of lacrimal drainage outflow at birth reveals obstruction rates that are much higher than those clinically observed in children with complaints of epiphora (12).

Almost all the pediatricians (86/90 [99\%]) in the current survey diagnosed CNLDO based on the clinical examination, 
likely due to the specific clinical manifestations. Although disappearance dye test has $90 \%$ sensitivity and $100 \%$ specificity, it can be difficult to perform in children due to the reaction to the instillation of the eyedrops (2). Hence, the clinical diagnosis is preferred by primary care physicians and ophthalmologists (6).

Massage was the initial treatment recommended by $95.5 \%$ of the pediatricians in the present survey. The lacrimal duct is the last to canalize during development (5) and the membrane can spontaneously open within several weeks of birth, before the onset of lacrimation. Often the child needs to be maintained with massage to empty the sac or hydrostatic massage (Crigler massage) $(6,13,17,18)$.

According to the pediatricians in the current survey, fewer than $50 \%$ of CNLDO cases required referral to an ophthalmologist due to failure of massage. The success rate with massage varies based on the age of the children, being greater in young children (19), but also depends on sac enlargement $(5,19,20)$.

Pediatric ophthalmologists routinely perform Crigler massage at the initial office visit in children younger than 6 months (13). However, massage can be the initial approach at all ages (17), even in children older than 13 months (11).

Massage is the initial approach for CNLDO among 63.9\% of oculoplastic surgeons from Latin America (10).

Notably, $14.65 \%$ of respondents in our survey did not recommend massage, compared to $2.8 \%$ of oculoplastic surgeons (10) and $55 \%$ of pediatric ophthalmologists (13). The differences between our study and previous publications could be due to the study sample of primary care pediatricians enrolled in our study who prefer to refer the patient or believe in prompt resolution.

Parents of children with CNLDO are taught how to do massage with frequency of 2 to 3 times daily $(60.43 \%$ of the caregivers who prescribe massage), as suggested by others (1).

Almost half of pediatricians $(47.56 \%)$ in our survey recommended continuation of the massage until the symptoms disappeared. Other studies (1) have instructed massage for 2 weeks after the disappearance of clinical signs. A study (10) of oculoplastic surgeons reported that $31 \%$ of respondents recommended massage until 6 months of age, which is similar to our result of $36.6 \%$ of respondents. However, $14.63 \%$ of pediatricians in our survey instructed the parents to perform a sac massage until 12 months of age, similar to a survey of oculoplastic surgeons (12\%) (10). Higher rates were reported in surveys of pediatric ophthalmologists (82\%) (13) and general ophthalmologists (84\%) (3).

As previously mentioned, the success rate of massage decreases with age, so pediatricians and oculoplastic surgeons prefer interventional treatment after 12 months of age.

Spontaneous resolution of CNLDO until 6 months of age was reported by $67.4 \%$ of pediatricians in our survey and by $66 \%$ of pediatricians from the Pediatric Eye Disease Investigator Group (21).

The outcomes of our survey indicate that $95.35 \%$ of $\mathrm{CN}$ LDO cases can be resolved around 1 year of age. The rates of spontaneous resolution of CNLDO are approximately $66 \%$ to $100 \%$ by 12 months of age $(2,14,21-25)$. With conservative management, CNLDO resolves in $70 \%$ of affected patients by 3 months and in $95 \%$ by 1 year $(4,5,22)$. The rate of sponta- neous resolution of CNLDO decreases in older children (10). However, spontaneous resolution can occur even in children beyond 12 months of age, which may mitigate the need for probing perhaps even beyond this time period (11).

Our study examined referral decision-making by asking physicians to record their reasons for referral. Effective communication between primary care and specialists is essential for successful comanagement when children are referred to specialty care (26). A referral is the result of a physicianpatient decision about the most appropriate course of action for management of a health problem (27).

The current survey indicates that $79.31 \%$ of pediatricians referred fewer than $50 \%$ of CNLDO cases to an ophthalmologist and $87.21 \%$ referred patients due to lack of improvement in symptoms and $10.47 \%$ due parent/guardian request. In general, the most common reason for a pediatrician to refer children is for advice on treatment ( $74.3 \%$ of cases) or parental request ( $15.7 \%$ of cases) (27).

The results of our study suggest that CNLDO management can be incorporated into a busy pediatrics practice and that pediatricians can significantly contribute to the overall ocular health of young children by identifying cases that warrant ophthalmic referral.

Half of the pediatricians in the current survey indicated that they have limited knowledge on etiology of CNLDO, and the other half responded that they have fair knowledge. However, only $35 \%$ received training in CNLDO. Nevertheless, they consider their knowledge on diagnosis and treatment of CNLDO as good. Almost all of the surveyed pediatricians $(92.2 \%)$ indicated that they would welcome further training on CNLDO. Based on these outcomes, we suggest that residency programs ensure that common conditions such as CNLDO be emphasized during training. Training will provide pediatricians with the skills necessary to expand their scope of practice as appropriate and determine appropriate cases for referral. This study has important implications for how physicians should be trained in the skills necessary for effective consultation and referral.

An advantage of our study is that we surveyed mostly pediatricians who routinely treat children with CNLDO. A limitation to our study is that around $40 \%$ of APACYL members responded to our survey, which may reflect that members with less clinical or surgical experience treating CNLDO were less inclined to take the survey. Although selection bias is an inherent flaw of survey studies, we have no reason to believe that the opinions of members who participated in the survey are different from those who did not. Another limitation is that the respondents were not balanced geographically. Although it is possible that the location and local practice patterns may influence referral choices, we were unable to assess this because we did not have the specific zip codes or knowledge of the pediatrician clinical context and cultural influences. Another important limitation is that these are self-reported practice preferences of pediatricians taking the time to respond to a survey questioning their attitudes toward CNLDO management and not the results of a randomized clinical trial. Therefore, interpretation should be limited to what is more commonly performed, without stating that less common behaviors are necessarily incorrect. 
In conclusion, this study highlights the importance of consensus among primary care pediatricians and ophthalmologists in management of CNLDO. It allows practitioners to compare their practice patterns regarding CNLDO management with those of their peers. Use of referral criteria may result in better treatment and management of CNLDO by pediatricians.

\section{Disclosures}

Financial support: No financial support was received for this submission.

Conflict of interest: None of the authors has conflict of interest with this submission.

\section{References}

1. Nucci P, Capoferri C, Alfarano R, Brancato R. Conservative management of congenital nasolacrimal duct obstruction. J Pediatr Ophthalmol Strabismus. 1989;26(1):39-43.

2. Takahashi Y, Kakizaki H, Chan WO, Selva D. Management of congenital nasolacrimal duct obstruction. Acta Ophthalmol. 2010;88(5):506-513.

3. Puvanachandra N, Trikha S, MacEwen CJ, Morris RJ, Hodgkins PR. A national survey of the management of congenital nasolacrimal duct obstruction in the United kingdom. J Pediatr Ophthalmol Strabismus. 2010;47(2):76-80.

4. Karti O, Karahan E, Acan D, Kusbeci T. The natural process of congenital nasolacrimal duct obstruction and effect of lacrimal sac massage. Int Ophthalmol. 2016;36(6):845-849.

5. Bhandari S, Sengupta S, Yadalla D, et al. Factors affecting treatment outcome in congenital nasolacrimal duct obstruction: $A$ retrospective analysis from South India. Indian J Ophthalmol. 2015;63(10):759-762.

6. Castelo Branco Neto E, Castelo Branco B, Cardoso CC, Carvalho RG, Mota E, Castelo Branco A. [Management of congenital nasolacrimal duct obstruction]. Arq Bras Oftalmol. 2009;72(1):75-78.

7. Zintl E, Deufrains A. [Contribution of the pediatrician in the early diagnosis of eye diseases. 2]. Kinderarztl Prax. 1989;57(6): 263-270.

8. Shields SR. Managing eye disease in primary care. Part 1. How to screen for occult disease. Postgrad Med 2000;108(5):69-72, 75-8.

9. Shields SR. Managing eye disease in primary care. Part 2. How to recognize and treat common eye problems. Postgrad Med. 2000;108(5):83-86, 91-96.

10. Schellini SA, Ariki CT, Sousa RL, Weil D, Padovani CR. Management of congenital nasolacrimal duct obstructionlatin american study. Ophthal Plast Reconstr Surg. 2013;29(5):389-392.

11. Schellini SA, Ferreira Ribeiro SC, Jaqueta E, Padovani $C R$, Padovani CR. Spontaneous resolution in congenital nasolac- rimal obstruction after 12 months. Semin Ophthalmol. 2007; 22(2):71-74.

12. Schellini SA, Hoyama E, Rocha MC, Rugollo A Jr, Padovani CR. Evaluation of lacrimal system outflow in the newborn by dye tests. Orbit. 2000;19(3):137-142.

13. Dotan G, Nelson LB. Congenital nasolacrimal duct obstruction: common management policies among pediatric ophthalmologists. J Pediatr Ophthalmol Strabismus. 2015;52(1):14-19.

14. Casady DR, Meyer DR, Simon JW, Stasior GO, Zobal-Ratner JL. Stepwise treatment paradigm for congenital nasolacrimal duct obstruction. Ophthal Plast Reconstr Surg. 2006;22(4):243-247.

15. MacEwen CJYJ, Young JD. Epiphora during the first year of life. Eye (Lond). 1991;5(Pt 5):596-600.

16. Young JD, MacEwen CJ. Managing congenital lacrimal obstruction in general practice. BMJ. 1997;315(7103):293-296.

17. Qian Y, Traboulsi El. Lacrimal sac compression, not massage. J Pediatr Ophthalmol Strabismus. 2009;46(4):252.

18. Sielicka D, Mrugacz M, Bakunowicz-Łazarczyk A. [Nasolacrimal duct disorders in children. Part I. Anatomy, physiology and clinical signs]. Klin Oczna. 2010;112(10-12):342-345.

19. Kushner BJ. The management of nasolacrimal duct obstruction in children between 18 months and 4 years old. J AAPOS. 1998;2(1):57-60.

20. Stolovitch C, Michaeli A. Hydrostatic pressure as an office procedure for congenital nasolacrimal duct obstruction. J AAPOS. 2006;10(3):269-272.

21. Pediatric Eye Disease Investigator Group. Resolution of congenital nasolacrimal duct obstruction with nonsurgical management. Arch Ophthalmol. 2012;130(6):730-734.

22. Kakizaki H, Takahashi Y, Kinoshita S, Shiraki K, Iwaki M. The rate of symptomatic improvement of congenital nasolacrimal duct obstruction in Japanese infants treated with conservative management during the 1 st year of age. Clin Ophthalmol. 2008;2(2):291-294

23. Paul TO, Shepherd R. Congenital nasolacrimal duct obstruction: natural history and the timing of optimal intervention. $J$ Pediatr Ophthalmol Strabismus. 1994;31(6):362-367.

24. Petersen RA, Robb RM. The natural course of congenital obstruction of the nasolacrimal duct. J Pediatr Ophthalmol Strabismus. 1978;15(4):246-250.

25. Nelson LB, Calhoun JH, Menduke H. Medical management of congenital nasolacrimal duct obstruction. Pediatrics. 1985; 76(2):172-175.

26. Stille CJ, McLaughlin TJ, Primack WA, Mazor KM, Wasserman RC. Determinants and impact of generalist-specialist communication about pediatric outpatient referrals. Pediatrics. 2006;118(4):1341-1349.

27. Forrest $C B$, Glade $G B$, Baker AE, Bocian AB, Kang M, Starfield $B$. The pediatric primary-specialty care interface: how pediatricians refer children and adolescents to specialty care. Arch Pediatr Adolesc Med. 1999;153(7):705-714. 